\title{
Direct Gram's stain of vaginal discharge as a means of diagnosing bacterial vaginosis
}

\author{
V. O. ROTIMI, Z. YAKUBU, O. O. ABUDU* and T. O. BANJO \\ Department of Medical Microbiology and Parasitology, and "Department of Obstetrics and Gynaecology, College of \\ Medicine, University of Lagos, PMB 12003, Lagos, Nigeria
}

\begin{abstract}
Summary. The diagnosis of bacterial vaginosis (BV) by direct gram-stained smear of vaginal discharge was evaluated in 90 consecutive patients. Vaginal secretions from 20 healthy female volunteers were also examined. All specimens were examined by culture for growth of Gardnerella vaginalis and Lactobacillus spp. Only $35(38.9 \%)$ of the patients with vaginal discharge satisfied the clinical diagnosis of BV. The vaginal gram-stained smear from all these 35 patients contained typical gardnerella morphotypes characteristic of BV and $G$. vaginalis was isolated from all of them as well as from a further 10 patients who did not satisfy the criteria for diagnosis of BV. The semi-quantitative assessment of gardnerella morphotypes was found to correlate positively with the semi-quantitative assessment of $G$. vaginalis growth by culture $(\mathrm{p}<0.0001)$. There was a strong inverse relationship between the quantity of lactobacillus and gardnerella morphotypes. Direct gram-staining of smears should facilitate the diagnosis of BV for clinicians with minimum need for confirmation by culture, particularly in a third world setting with few or no laboratory facilities.
\end{abstract}

\section{Introduction}

Since the initial report by Gardner and Dukes in $1955,{ }^{1}$ non-specific vaginosis (NSV) has become accepted as an important clinical entity, particularly in gynaecological practice. ${ }^{2-5}$ Although the aetiology and pathogenesis of NSV have not been unequivocally defined, there is evidence for its association with the isolation of Gardnerella vaginalis ${ }^{1,5,6}$ and possibly some other micro-organisms including anaerobic bacteria $^{3,5}$ such as Bacteroides spp., anaerobic cocci and the comma-shaped rods now identified as Mobiluncus spp. ${ }^{7}$ As a result, the condition has become known as bacterial vaginosis (BV).

Spiegel et al..$^{7,8}$ described characteristic appearances in gram-stained smears of vaginal discharge from women with BV; these were a predominance of gardnerella morphological types along with other small gram-negative rods and gram-positive cocci, in contrast to a predominance of lactobacillus morphological types in vaginal secretions from normal, healthy women. A similarly characteristic appearance is seen in wet films of vaginal discharge, in which vaginal epithelial cells coated with small, pleomorphic bacilli are prominent. These cells are known as "clue cells"., 8

Recently, gas-liquid chromatography $(\mathrm{GLC})^{8}$ and thin-layer chromatography have been used for the detection of bacterial organic acid metabolites and diamines respectively and have been demonstrated to exhibit patterns characteristic for the diagnosis of

Received 20 April 1990; revised version accepted 27 Nov. 1990.
BV. ${ }^{9}$ However, the equipment needed for these tests is generally unavailable to many laboratories in the developing countries. Furthermore, the isolation of $G$. vaginalis or anaerobic bacteria, or both, from women without bacterial vaginosis precludes the use of culture alone in the laboratory diagnosis of $\mathrm{BV} .{ }^{10}$

Thus there are no simple tests that enable the medical microbiologist to aid the clinician in confirming the diagnosis of BV. Various investigators have described an appearance in gram-stained smears of vaginal fluid indicative of $B V ;, 8,11-13$ normal vaginal fluid contained only lactobacillus morphotypes whereas fluid from patients with clinically diagnosed BV had mainly gram-negative bacteria resembling $G$. vaginalis with no lactobacillus morphotypes.

However, although reports suggest that a simple Gram's stain may be used for the diagnosis of BV, only one has so far demonstrated the value of this method. ${ }^{8}$ In this study we compared the appearance in gram-stained smears of va lal discharge from BV patients and healthy controls with a composite of clinical criteria presently used for the diagnosis of BV in our location, and correlated this with the isolation of G. vaginalis. The value of Gram's stain alone for the diagnosis of $\mathrm{BV}$ in our hospital is thereby assessed.

\section{Materials and methods}

\section{Subjects}

Ninety pregnant and non-pregnant women with vaginal discharge attending the Obstetrics and Gynae- 
cology Clinics of the Lagos University Teaching Hospital (LUTH) between March and May, 1989, were studied. There was no attempt to select any particular gestational age of the pregnant women. Twenty normal, healthy volunteers without vaginal discharge were also studied as a control group. The non-pregnant women were in the proliferative phase of their menstrual cycle.

The age, parity and marital status of the patients and the gestational age of the pregnancies were recorded. Subjects on oral antimicrobial therapy or any form of vaginal medication were excluded from the study. All the patients were interviewed and examined by the same gynaecologist (OOA) and they were evaluated by standardised interview with questions concerning the use of contraceptives and duration of their symptoms.

\section{Specimens}

The posterior and lateral vaginal fornices and cervix were sampled by high vaginal (HVS) and cervical (CS) swabs after exposing the cervical os with a sterile, unlubricated Cusco's speculum; duplicate HVS and CS were collected in Amies' transport medium (Oxoid). The characteristics of the discharge such as quantity, colour and smell were recorded and the $\mathrm{pH}$ of the discharge was determined directly with $\mathrm{pH}$ indicator paper. An immediate fishy odour on addition of $1-2$ drops of $\mathrm{KOH} 10 \%$ was noted as a positive amine test. ${ }^{12}$

A diagnosis of bacterial vaginosis was made in this study when three of the following characteristics were detected: (i) vaginal $\mathrm{pH} \geqslant 5 \cdot 0$; (ii) thin homogenous discharge; (iii) presence of "clue" cells; (iv) positive amine test. The subject was considered normal if three of these criteria were not met and neither yeasts nor trichomonads were detected microscopically.

\section{Microbiological examination}

The sets of specimens in Amies' transport medium were processed within $2 \mathrm{~h}$ of the time of collection; one of each pair was used to prepare smears for microscopy, the other for inoculation of culture media.

Microscopic examination. A wet preparation and a gram-stained smear from each sample were examined microscopically using a $\times 40$ objective for the wet film and a $\times 100$ oil-immersion objective for the gramstained smear. The numbers of "clue" cells and normal epithelial cells were estimated. In wet films, lactobacillus and gardnerella morphotypes were estimated semi-quantitatively as previously described according to the following scheme; $1^{+}, 1$ /field $; 2^{+}, 1-5 /$ field $; 3^{+}$, $6-30$ /field ; $4^{+}, 30$ /field. Gram-stained vaginal smears were assessed for the presence of bacterial vaginosis by the criteria of Spiegel et al. ${ }^{8}$

Bacterial culture. Three media were used for primary cultures: selective Brain Heart Infusion Agar (SBHIA) for G.vaginalis, comprising Brain Heart Infusion Agar
(Oxoid) with expired human blood $5 \%$, neomycin 75 $\mathrm{mg} / \mathrm{L}$ and colistin $10 \mathrm{mg} / \mathrm{L} ;{ }^{14}$ selective chocolate agar (SCA) for Neisseria gonorrhoeae, comprising Blood Agar Base No. 2 (Oxoid) with expired human blood $10 \%$, vancomycin $3 \mathrm{mg} / \mathrm{L}$ and colistin $7.5 \mathrm{mg} / 1$; and Sabouraud Dextrose Agar (Oxoid) for the cultivation of Candida spp. The swabs from Amies' transport medium were inoculated on to the media by standard plating techniques. ${ }^{15}$ For semi-quantitative cultures of G. vaginalis and lactobacilli, a four-streak zonal plating method was used. ${ }^{16}$ Plates of Sabouraud's dextrose agar were incubated in air at $37^{\circ} \mathrm{C}$; other plates were incubated in $\mathrm{CO}_{2} 5-10 \%$ in a candle extinction jar at $37^{\circ} \mathrm{C}$ and observed every $24 \mathrm{~h}$ for at least 3 days.

Identification of isolates. $N$. gonorrhoeae and $C$. albicans were identified by standard methods. ${ }^{17}$ Isolates that produced tiny $\beta$-haemolytic colonies on SBHIA, were gram-negative or gram-variable coccobacilli in gram-stained smears, and were catalasenegative were identified presumptively as $G$. vaginalis. ${ }^{1,14}$ Their identity was confirmed by a battery of biochemical tests including their ability to ferment maltose and a negative oxidase test. ${ }^{14}$ Typical large gram-positive bacilli from non-haemolytic colonies were identified presumptively as lactobacilli.

Semi-quantitative assessment of growth. Growth of $G$. vaginalis and lactobacilli was assessed semiquantitatively ${ }^{9}$ on SBHIA according to an arbitrary scale of $1^{+}-4^{+}$in which $1^{+}=\leqslant 10$ colonies in the inoculum pool $; 2^{+}=>10$ colonies in the first inoculation zone and $<10$ colonies in the second zone $; 3^{+}=$ luxuriant growth in the inoculum pool, $>10$ colonies in the second zone and $\leqslant 10$ colonies in the third zone; $4^{+}=$luxuriant growth in pool, second and third zones, and $>10$ colonies in the fourth zone.

\section{Statistical analysis}

The data obtained were subjected to statistical analysis by Fisher's exact test.

\section{Results}

The general clinical features of the 90 patients and 20 healthy controls are summarised in table I. A vaginal $\mathrm{pH}$ of $\geqslant 5.0$ was recorded in $86(95.6 \%)$ of the 90 patients but in only two ( $10 \%$ ) out of the 20 normal women. Moderate to profuse discharge was present in $81(90 \%)$ out of the 90 patients and was malodorous in $68(75.6 \%)$. The presence of "clue" cells was recorded in $37(41.1 \%)$ of the patients; the amine test was positive in only five cases $(5.5 \%)$. Thirty-five $(38.9 \%)$ of the 90 patients investigated were diagnosed clinically as having BV by any three of the diagnostic criteria described earlier.

Table II shows the vaginal microflora as assessed by microscopic examinations of a gram-stained smear of vaginal discharge from patients diagnosed clinically 
Table I. Characteristics of patients with vaginal discharge and healthy controls

\begin{tabular}{l|cc}
\hline \multirow{2}{*}{ Characteristic } & \multicolumn{2}{|c}{ Number of women in group } \\
\cline { 2 - 3 } & $\begin{array}{c}\text { with vaginal } \\
\text { discharge }(\mathrm{n}=90)\end{array}$ & $\begin{array}{c}\text { with no } \\
\text { discharge }(\mathrm{n}=20)\end{array}$ \\
\hline $\mathrm{pH}$ estimation & & \\
3 & 0 & 0 \\
4 & 4 & 18 \\
5 & 6 & 2 \\
6 & 23 & 0 \\
7 & 45 & 0 \\
8 & 12 & 0 \\
Amount of discharge & & 0 \\
Small & 9 & 0 \\
Moderate & 57 & 0 \\
Profuse & 24 & 0 \\
Positive amine test & 5 & 0 \\
Malodour & 68 & 0 \\
"Clue cells" present & 37 & 0 \\
Pus cells present & 3 & \\
& &
\end{tabular}

Table II. Vaginal microflora in patients with and without clinically diagnosed BV as determined by microscopy of a gram-stained smear of vaginal discharge

\begin{tabular}{l|ccl}
\hline \multicolumn{1}{c|}{$\begin{array}{c}\text { Morphotypes of } \\
\text { organisms seen }\end{array}$} & \multicolumn{2}{|c}{ Clinical diagnosis } & \\
\cline { 2 - 3 } & $\begin{array}{c}\mathrm{BV} \\
(\mathrm{n}=35)\end{array}$ & $\begin{array}{c}\text { non-BV } \\
(\mathrm{n}=55)\end{array}$ & $\mathrm{p}$ value \\
& & & \\
\hline $\begin{array}{l}\text { Gram-positive cocci } \\
\text { Gram-negative coccobacilli }\end{array}$ & 26 & 5 & $\mathrm{p}<0.005$ \\
$\begin{array}{l}\text { Lactobacillus morphotype } \\
0-2^{+}\end{array}$ & 32 & 0 & $\mathrm{p}<0.005$ \\
$3^{+}-4^{+}$ & 0 & 9 & $\mathrm{p}<0.005$ \\
$\begin{array}{l}\text { Gardnerella morphotype } \\
\text { Curved rods }\end{array}$ & 35 & 7 & $\mathrm{p}<0.005$ \\
& 17 & 0 & $\mathrm{p}<0.005$ \\
& & & \\
\hline
\end{tabular}

as having BV and controls without BV. For all 35 patients with a clinical diagnosis of $\mathrm{BV}$, the gramstained appearance was consistent with BV. The gardnerella morphotype was observed in smears from all 35 patients, which was significantly higher than their presence in smears from seven $(12.7 \%)$ of 55 patients reported clinically as non-BV $(p<0.005)$. The lactobacillus morphotype was consistently absent, or present in only low numbers $\left(1^{+}-2^{+}\right)$, in all patients diagnosed clinically as having $\mathrm{BV}$; such low numbers were recorded in only nine $(16.4 \%)$ of 55 non-BV patients $(\mathrm{p}<0.005)$. Heavy counts of lactobacilli $\left(3^{+}\right.$ $4^{+}$) were recorded consistently in the absence of gardnerella morphotypes in $46(83.6 \%)$ of the 55 nonBV patients.

Gram-positive cocci, gram-negative coccobacilli and curved rods were reported in significantly more patients with than without a clinical diagnosis of BV $(p<0.005)$. The semi-quantiative assessment of gardnerella morphotype was positively associated with the semi-quantitative assessment of growth of $G$. vaginalis $(\mathrm{p}<0.0001)$ (table III). Gram-stained smear and culture were simultaneously positive (42) or negative (34) in $76(84.4 \%)$ of the 90 cases.

A strong inverse relationship between the quantitative assessment of lactobacillus and gardnerella morphotypes was found in the 90 gram-stained vaginal smears studied ( $p<0.0001)$ (table IV). As the gardnerella morphotypes increased from 0 to $4^{+}$, the lactobacillus morphotypes were observed to diminish from $4^{+}$to 0 . However, there were seven cases in which the gram-stained vaginal smears showed more gardnerella morphotypes than lactobacillus morphotypes but they were not consistent with a diagnosis of $\mathrm{BV}$ because other mixed coccabacilliary flora were not present; furthermore, these cases were not diagnosed clinically as $\mathrm{BV}$.

\section{Discussion}

In this study, the diagnosis of $\mathrm{BV}$ in patients by examination of a gram-stained smear was evaluated by standard microbiological and clinical criteria. All 35 cases with a clinical diagnosis of BV had the same diagnosis made from the gram-stained smear alone, thus indicating a high positive correlation between the two.

An increased prevalence of gram-negative rods and gram-positive cocci was observed in the smears obtained from patients with $\mathrm{BV}$, a finding consistent with earlier reports of an increase in the prevalence

Table III. Comparison of semi-quantitative isolation of $G$. vaginalis on SBHIA versus semi-quantitative counts of gardneralla morphotypes on gram-stained smears of vaginal discharge

\begin{tabular}{|c|c|c|c|c|c|}
\hline \multirow{2}{*}{$\begin{array}{l}\text { Gardnerella } \\
\text { morphotype } \\
\text { score in smear }\end{array}$} & \multicolumn{5}{|c|}{$\begin{array}{c}\text { Number of patients with } G . \text { vaginalis scores on } \\
\text { culture }\end{array}$} \\
\hline & 0 & $1+$ & $2+$ & $3+$ & $4+$ \\
\hline 0 & 34 & 6 & 4 & 0 & 0 \\
\hline $1^{+}$ & 0 & 0 & 0 & 0 & 0 \\
\hline $2^{+}$ & 3 & 0 & 0 & 7 & 5 \\
\hline $3^{+}$ & 1 & 0 & 0 & 3 & 2 \\
\hline $4^{+}$ & 0 & 0 & 0 & 6 & 19 \\
\hline
\end{tabular}

Table IV. Clinical diagnosis of BV in relation to gram-stain quantitation of lactobacillus and gardnerella morphotypes

\begin{tabular}{c|rcccc}
\hline & \multicolumn{5}{c}{$\begin{array}{c}\text { Lactobacillus } \\
\begin{array}{c}\text { morphotype } \\
\text { score }\end{array}\end{array}$} \\
\cline { 2 - 6 } & 0 & $1^{+}$ & $2^{+}$ & $3^{+}$ & $4^{+}$ \\
\hline 0 & 0 & 0 & $2(2)$ & 0 & $17(15)$ \\
$1^{+}$ & 0 & 0 & $1(1)$ & $5(5)$ & $10(8)$ \\
$2^{+}$ & 4 & 0 & 0 & 3 & $4(4)$ \\
$3^{+}$ & 5 & 0 & 0 & 1 & 1 \\
$4^{+}$ & 36 & 0 & 0 & 1 & 0 \\
\hline
\end{tabular}

The numbers in parentheses indicate the numbers of patients with BV. 
and quantity of Bacteroides sp. and anaerobic grampositive cocci in the vaginal fluid of women with BV. ${ }^{18,22}$ Furthermore, the presence of curved rods was also positively correlated with the diagnosis of BV. The investigators have noted the presence of motile curved rods ${ }^{19,20,22}$ identified as Mobiluncus spp. although their role in BV is not clear.

Other studies have seemed to suggest that a gramstained smear of vaginal discharge is more useful than semi-quantitative culture of $G$. vaginalis for the diagnosis of $\mathrm{BV},{ }^{8,22}$ an observation supported by our findings; none of the 10 specimens for which the gramstained smear gave a negative result but the culture for $G$. vaginalis was positive had $>2^{+}$score for $G$. vaginalis on semi-quantitative assessment. The four specimens with a positive gram-stain result for gardnerella morphotypes and a negative culture for $G$. vaginalis may have contained strains of $G$. vaginalis that failed to grow under our microaerophilic conditions. $^{8}$

The detection of $G$. vaginalis either by Gram's stain or by culture alone cannot be recommended as a method for the diagnosis of BV because the organism is often a constituent of the normal vaginal flora. ${ }^{9}$ In this study, of the 42 women from whom $G$. vaginalis was isolated, seven did not have a clinical diagnosis of BV. This lack of value of vaginal culture for $G$ vaginalis in the diagnosis of $\mathrm{BV}$ has been reported, ${ }^{18,21}$ although there is a highly significant association between BV and the presence of $G$. vaginalis. ${ }^{21}$ Our experiences in this study conform with those of Amsel

\section{References}

1. Gardner HL, Dukes CD. Hemophilus vaginalis vaginitis. Am J Obstet Gynecol 1955; 69: 962-976.

2. Abudu OO, Odugbemi TO, Agboola A. Corynebacterium vaginale and vaginitis. W Afr Med J 1982; 1: 19-20.

3. Spiegel CA, Amsel R, Eschenbach D, Schoenknecht F, Holmes KK. Anaerobic bacteria in nonspecific vaginitis. $N$ Engl J Med 1980; 303: 601-607.

4. Phillips I, Taylor E. Anaerobic curved rods in vaginitis. Lance $1982 ; 1: 221$

5. Rotimi VO, Kayode Y, Oji CO, Dosunmu-Ogunbi O. The bacterial flora of non-specific vaginitis. Afr J Sex Trans Dis $1984 ; 2: 34-37$

6. Rotimi VO, Oji GO. Gardnerella vaginalis in non-specific vaginitis: its occurrence and antimicrobial susceptibility. Afr J Clin Microbiol 1984; 1: 47-54.

7. Spiegel CA, Eschenbach DA, Amsel R, Holmes KK. Curved anaerobic bacteria in bacterial (nonspecific) vaginosis and their response to antimicrobial therapy. $J$ Infect Dis 1983 148: 817-822.

8. Spiegel CA, Amsel R, Holmes KK. Diagnosis of bacterial vaginosis by direct gram stain of vaginal fluid. $J$ Clin Microbiol $1983 ; 18$ : 170-177.

9. Chen KCS, Amsel R, Eschenbach DA, Holmes KK. Biochemical diagnosis of vaginitis: determination of diamines in vaginal fluid. $J$ Infect Dis $1982 ; 145$ : $337-345$.

10. Bump RC, Buesching WJ. Bacterial vaginosis in virginal and sexually active adolescent females: evidence against exclusive sexual transmission. Am J Obstet Gynecol 1988; 158: 935-939.

11. Dunkelberg WE. Diagnosis of Hemophilus vaginalis vaginitis by gram-stained smears. Am J Obstet Gynecol 1965; 91 : 998-1000.

12. Balsdon MJ, Taylor GE, Pead L, Maskell R. Corynebacterium vaginale vaginitis: a controlled trial of treatment. Lancet $1980 ; 1: 501-504$ et $a .^{21}$ and Spiegel et al. ${ }^{8}$ who found that there is a high positive correlation between microscopy and clinical diagnosis for the detection of $\mathrm{BV}$. The inverse relationship between the presence and concentration of gardnerella and lactobacillus morphotypes in the gram-stained smears observed in this study is in agreement with their reports.

Although the presence of "clue" cells in a wet preparation of vaginal discharge correlated very well (table I) with the clinical diagnosis of $\mathrm{BV}$, the advantages of a gram-stained smear over "clue" cell determination in a wet film in the diagnosis of BV include standardised reading by a microbiologist, applicability for screening large populations, and the fact that gram-stained smears can be stored and transported for batch reading if so desired. ${ }^{16}$

This study is certainly not the first attempt to diagnose BV by microscopic examination of vaginal discharge but it is the first report of its kind for a tropical African setting, and demonstrated the practicability of using microscopy for confirming the diagnosis of $\mathrm{BV}$ in a way that correlates well with clinical and microbiologic data as indicated in previous reports ${ }^{8,21}$ for developed countries. The methods of microscopy used in this study for the diagnosis of BV would be suitable for a clinical microbiology setting in developing countries or where sophisticated methods such as gas-liquid and thin-layer chromatography are not readily accessible, and could be used to complement or confirm the clinician's evaluation of patient's with abnormal vaginal discharge.

13. Pheifer TA, Forsyth PS, Durfee MA, Pollock HM, Holmes KK. Nonspecific vaginitis: role of Haemophilus vaginalis and treatment with metronidazole. N Engl J Med 1978; 298: $1429-1434$

14. Oji GO, Rotimi VO. Gardnerella vaginalis in non-specific vaginitis: isolation and identification. Afr J Clin Microbiol $1984 ; 1: 39-46$

15. Gillies RR, Dodds TC. Bacteriology illustrated. Edinburgh, Churchill Livingstone. 1976

16. Eschenbach DA, Hilliers S, Critchlow C, Stevens C, DeRonen T, Holmes KK. Diagnosis and clinical manifestations of bacterial vaginosis. Am J Obstet Gynecol 1988; 158: 819 828.

17. Lennette EH, Balows A, Hausler WJ, Shadomy HJ (eds) Manual of clinical microbiology, 4th edn. Washington, DC, American Society for Microbiology. 1985.

18. Totten PA, Amsel RA, Hale J, Piot P, Holmes KK. Selective differential human blood bilayer media for isolation of Gardnerella (Haemophilus) vaginalis. J Clin Microbiol 1982; 15: 141-147.

19. Popp $W$. The diagnosis and treatment of mixed anaerobic vaginal discharges. Geburtshilfe Frauenheilkd 1977; 37 $432-437$.

20. Thomason JL, Schreckenberger PC, Spellacy WN, Ruff LJ, LeBeau LJ. Clinical and microbiological characterization of patients with nonspecific vaginosis associated with motile, curved anaerobic rods. J Infect Dis 1984; 149: 801 809.

21. Amsel R, Totten PA, Spiegel CA, Chen KCS, Eschenbach D, Holmes KK. Nonspecific vaginitis : diagnostic criteria and microbial and epidemiologic associations. Am J Med 1983; 74: 14-22.

22. Spiegel CA, Amsel R, Eschenbach D, Schoenknecht F, Holmes $\mathrm{KK}$. Anaerobic bacteria in nonspecific vaginitis. $N$ Engl Med 1980; 303: 601-607. 